\title{
XP \& Skills: gamificando o processo de ensino de introdução a programação
}

\author{
André Luiz de Souza Brito ${ }^{1}$, Charles Andryê Galvão Madeira ${ }^{1}$ \\ ${ }^{1}$ Instituto Metrópole Digital - Universidade Federal do Rio Grande do Norte (UFRN) \\ Natal - Rio Grande do Norte - Brasil \\ andreeimd.ufrn.br, charleseimd.ufrn.br
}

\begin{abstract}
Introductory programming classes usually present high levels of retention and evasion. This paper explores the use of a gamified process to address the motivation issue of students in these classes. A conceptual model based on Werbach framework and the Design-thinking methodology is proposed, with a description of the phases developed in the project (immersion, ideation and prototyping). The results gathered from the execution of the methodology in a real class demonstrate its potential and give us leads to areas of improvement.
\end{abstract}

Resumo. Disciplinas introdutórias de programação ofertadas por cursos de tecnologia no país apresentam altas taxas de reprovação, retenção e evasão. Este artigo explora o uso da gamificação no tratamento do problema da motivação e participação de alunos cursando este tipo de disciplina. Um modelo conceitual é proposto baseado no framework de gamificação de Werbach e na metodologia de Design Thinking. Os resultados inicialmente obtidos pela execução do processo gamificado no contexto de uma turma real de graduação demonstram o grande potencial da sua aplicação e apontam diversas possibilidades de melhorias.

\section{Introdução}

Historicamente, disciplinas de introdução a programação apresentam um alto índice de reprovação, retenção e evasão, constituindo um grande problema para os cursos da área tecnológica nos mais diversos níveis de educação. De acordo com [Gomes et al. 2008], o principal problema ocorre na dificuldade que uma porcentagem significativa dos alunos tem em conseguir mapear um problema real para uma solução computacional utilizando os comandos e estruturas vistos no decorrer do curso. Existem diversos fatores que contribuem para essa situação: o aprendizado da programação requer bastante esforço em exercícios práticos e tempo de dedicação. Além disso, falta uma base matemática adequada e habilidade na resolução de problemas, deficiências oriundas de uma formação básica que não desenvolve essas competências nos alunos. Já [Jenkins 2001] acrescenta que a própria motivação do aluno em aprender a programação está diretamente relacionada com a sua capacidade de visualizar o impacto e importância daquele conhecimento na sua formação.

Uma das implicações desse cenário é a rápida desmotivação do aluno com a área de programação, ocasionando seu atraso no curso. Diante desta situação, torna-se imprescindível buscar formas de entender melhor as causas do problema e investigar possíveis soluções que possam remediá-lo de forma efetiva. 
Um dos possíveis caminhos existentes na literatura é o da Gamificação [Deterding et al. 2011] no qual busca-se motivar, engajar e, idealmente, modificar positivamente o comportamento de um determinado público-alvo através do uso de recursos e elementos do mundo dos jogos. Neste trabalho, a gamificação é usada como uma ferramenta motivacional para os alunos de disciplinas de programação. Assim, partimos da hipótese de que ao motivarmos e engajarmos os alunos com as suas tarefas, os resultados apresentados pelos mesmos poderão ser bem melhores dos que os obtidos até o presente momento através dos métodos tradicionais de ensino.

O documento está organizado da seguinte forma: na segunda seção serão apresentados trabalhos relacionados com a aplicação da gamificação em ambientes educacionais e de aprendizado. A terceira seção fará uma explanação sobre o framework de gamificação de Werbach [Werbach and Hunter 2012] e a metodologia de Design Thinking [Brown 2010], bases para o desenvolvimento da proposta apresentada nesse artigo. Na quarta seção será introduzida a nossa proposta de modelo conceitual de gamificação para disciplinas de programação, detalhando as fases de imersão e ideação definidas pela metodologia de Design Thinking. Na quinta seção serão apresentados os primeiros resultados obtidos com a experimentação (prototipação e validação) do modelo proposto em uma turma da disciplina de introdução às técnicas de programação de graduação em Tecnologia da Informação. Por fim, o texto será concluído elencando as possíveis direções que o trabalho poderá tomar a partir dos primeiros resultados obtidos.

\section{Trabalhos Relacionados}

Atualmente existe uma boa quantidade de trabalhos sendo feitos sobre a gamificação de ambientes de aprendizado (salas de aula, ambientes virtuais) e o impacto percebido na utilização dos métodos lúdicos para motivação e engajamento dos alunos. Em [Hamari et al. 2014], encontra-se uma revisão geral de vários trabalhos envolvendo gamificação, além de uma taxonomia dos métodos e resultados. Como pode ser constatado a partir deste artigo, os esforços dispensados para entender os verdadeiros ganhos de modelos gamificados são intensos. Muitos destes modelos são focados no uso de três elementos clássicos dos jogos conhecidos como o trio PBL (Points, Badges \& Leaderboards): Pontos (pontuação que o jogador recebe ao executar determinadas ações ou comportamentos incentivados pelo processo), Medalhas (marcos ou conquistas que são dados ao jogador como uma forma de reconhecer que ele atingiu um patamar ou status desejado), Ranking de Líderes (classificação do jogador com relação aos outros jogadores envolvidos no processo usada como métrica de comparação de sua performance).

Em [Denny 2013] mostra-se a utilização de metodologias baseadas em medalhas de forma a motivar o usuário ofertando uma recompensa pela sua atuação. Em [Domínguez et al. 2013] também é utilizado o elemento de ranking de líderes, apresentando os usuários/jogadores que tiveram a melhor performance dentro dos parâmetros estabelecidos pelo estudo. Em [Cheong et al. 2013] apresenta-se uma metodologia fortemente orientada a pontos e ao feedback para o usuário, onde utiliza-se um sistema gamificado para que o aluno exercite seus conhecimentos sobre assuntos específicos.

Em [Borges et al. 2013], uma revisão sistemática é feita especificamente na área de gamificação na educação. Os autores realizaram uma categorização de diversos trabalhos considerando vários critérios: ano e forma de publicação, público-alvo da 
gamificação, objetivo da pesquisa e problemáticas abordadas pelos trabalhos. Já em [Falcão et al. 2014] e [da Rocha Seixas et al. 2014] são investigados os impactos do uso de sistemas gamificados em sala de aula.

\section{Framework para Gamificação}

A proposta de gamificação apresentada nesse trabalho será baseada no framework apresentado por Werbach em [Werbach and Hunter 2012]. Este framework adota um processo de gamificação a partir da aplicação de uma metodologia de Design Thinking [Brown 2010].

Design Thinking é um processo focado no entendimento da problemática de forma aprofundada, para só então se propor soluções que a resolvam de forma efetiva. Isso é alcançado através de uma imersão profunda no contexto do problema, buscando as verdadeiras raízes da questão investigada. A partir daí projeta-se uma solução que possa resolvê-la com eficácia.

Existem três etapas que compõem o Design Thinking:

- Imersão: estudo aprofundado do problema junto ao público-alvo;

- Ideação: geração de várias ideias para compor possíveis soluções para a problemática;

- Prototipação: teste e validação das soluções propostas junto ao público-alvo.

Embasado nessa metodologia, Werbach propôs um framework de gamificação contendo seis etapas distintas que representam passos a serem tomados na construção de uma solução, fortemente centrada no entendimento do problema por parte da equipe proponente. Essas etapas são:

- Definir objetivos de negócios: entendimento do problema que se quer resolver e qual o ambiente em que ele ocorre;

- Definir metas: definição dos objetivos desejados a partir do que se almeja com a gamificação do sistema;

- Descrever jogadores: conhecer as pessoas envolvidas no processo e que interagem com o problema no dia a dia, ou seja, as peças centrais do processo de gamificação;

- Definir ciclos de atividades: após o entendimento do problema, deve-se pensar nas ações que poderão ser efetivadas no sistema para resolver o problema.

- Divertir os jogadores: a diversão é um dos elementos mais importantes em jogos e sistemas gamificados pois permite estimular a participação dos envolvidos no processo;

- Disponibilizar as ferramentas apropriadas: após definida uma proposta de solução, deve-se escolher as técnicas, modelos ou ferramentas adequadas para colocá-las em prática.

Esse ciclo de etapas deve ser continuamente testado e repetido até que uma solução madura e aceitável seja alcançada.

\section{Modelo Gamificado para Disciplinas de Programação}

Tendo como base o framework descrito na seção anterior, nesta seção serão apresentadas as etapas seguidas para desenvolver a nossa proposta de modelo gamificado para disciplinas de programação. 
CBIE-LACLO 2015

Anais dos Workshops do IV Congresso Brasileiro de Informática na Educação (CBIE 2015)

\subsection{Imersão}

Iniciamos a etapa de imersão com o objetivo de conhecer melhor o público-alvo (alunos). Para isso, duas perguntas precisavam ser claramente respondidas: qual a expectativa de mudanças de comportamento dos participantes do processo com o uso da gamificação? Qual o perfil das pessoas que participam do processo?

Como já mencionado anteriormente, a resposta a primeira pergunta consiste em melhorar a motivação e participação dos alunos na disciplina. A expectativa é que eles se interessem mais pelo assunto, efetuem os exercícios propostos e, consequentemente, apresentem um melhor desempenho acadêmico, aumentando o nível de aprovação geral da turma. Também espera-se que a média das notas da turma se eleve em comparação às notas dos anos anteriores. Além dessas métricas quantitativas, espera-se também um retorno direto do aluno, através de uma descrição da experiência de ter cursado uma disciplina gamificada e que tipo de influência os elementos adicionados na disciplina tiveram sobre o caráter motivacional e de divertimento ao longo do curso.

Para responder a segunda pergunta, precisávamos traçar o perfil dos alunos. Para isto, foi aplicado um questionário em sala no primeiro dia de aula para coletar as seguintes informações:

- O aluno trabalha?

- O aluno tem experiência com programação de computadores?

- O aluno já cursou essa disciplina anteriormente?

- O aluno tem maiores dificuldades em acompanhar disciplinas na universidade?

- O aluno gosta de jogos? Gostaria de ter elementos de jogos nessa disciplina?

O questionário foi respondido de forma anônima por 31 alunos. O resultado das respostas apresentou uma turma bastante heterogênea: $51 \%$ dos alunos trabalham, $45 \%$ já possui alguma experiência com programação, $70 \%$ já cursaram a disciplina de introdução às técnicas de programação em semestres anteriores, não concluindo-a por desistência ou reprovação. Quanto as dificuldades enfrentadas, as três mais citadas foram: estudo diário do conteúdo dado (45\%), disposição para resolução de exercícios (26\%) e cansaço devido ao dia de trabalho (22\%). Por último, $93 \%$ dos alunos demonstraram interesse em jogos e no uso de elementos de jogos dentro da sala de aula.

Dentro desse panorama, observa-se que temos um bom argumento para a aplicação de uma metodologia gamificada: os alunos têm uma forte predisposição à adoção de elementos de jogos na disciplina. Como citado em [Schell 2014], a voluntariedade do participante é um ponto fundamental em qualquer processo ou experimento que busque o uso de jogos e seus elementos.

O questionário também permitiu a segmentação da turma em quatro perfis genéricos (Newbie, Top Dogs, Workaholics e All-Nighters), baseados em experiência de trabalho e tempo de dedicação disponível para o curso. Esses perfis, listados na Tabela 1, passaram a orientar o processo de decisão sobre os aspectos trabalhados na turma para gamificação da disciplina. Embora tanto o grupo dos All-Nighters quanto o dos Workaholics trabalhem durante o dia, os Workaholics trabalham com computação e possuem experiência com programação, tendo menos dificuldades no decorrer do curso. Já os AllNighters são alunos que trabalham em áreas afins à computação, e a falta de experiência os 
Tabela 1. Classificação dos alunos da disciplina em formato de personas

\begin{tabular}{l|l|l|l} 
Grupo & $\begin{array}{l}\text { Experiência com } \\
\text { Programação }\end{array}$ & $\begin{array}{l}\text { Tempo } \\
\text { Dedicação }\end{array}$ & Representação \\
\hline Newbie & Não possui & Não trabalha & $20 \%$ \\
Top Dogs & Possui & Não trabalha & $23 \%$ \\
Workaholics & Possui & Trabalha & $27 \%$ \\
All-Nighters & Não possui & Trabalha & $30 \%$
\end{tabular}

torna um grupo de risco no processo de acompanhamento da disciplina devido ao tempo limitado de estudo fora da sala de aula.

Como métricas de comparação da metodologia gamificada com a tradicional, será tomado por base o percentual de aprovação de alunos na disciplina. Espera-se que com a aplicação da metodologia gamificada esse percentual fique acima do obtido sem o uso da metodologia. Além disso, espera-se que tanto o número de alunos aprovados de forma direta (sem necessidade de fazer uma prova de reposição) quanto a nota média final obtida pelos alunos, aumentem em relação a turmas passadas.

\subsection{Ideação}

O primeiro passo após o levantamento do perfil da turma e estabelecimento das métricas foi elencar os pontos ou dificuldades que uma metodologia gamificada poderia modificar. Pela experiência coletada com outros professores e também percebida no questionário aplicado, um dos pontos críticos do semestre ocorre após as primeiras avaliações: se o aluno obtém uma nota baixa, ele não tem uma perspectiva de recuperação, desestimulase e acaba por desistir da disciplina. Logo, um dos focos do modelo de gamificação proposto consiste em trabalhar a percepção de como o aluno enxerga a nota: deseja-se mudar o entendimento do aluno de que ele "perde" a nota, criando uma mentalidade de que a nota é conquistada. Apesar de ser uma mudança simples de perspectiva, existe uma possibilidade real que isso melhore a motivação dos alunos, sendo uma tentativa inicial de estabelecer uma motivação intrínseca pela disciplina (a conquista do conhecimento).

Outro aspecto observado é a temporalidade da avaliação: passada uma avaliação, o aluno sente que a nota é imutável e que aquele estágio está perdido. Ao invés de julgar o aluno em períodos determinados e manter a nota estática, foi definido um esquema no qual o aluno é avaliado em função das habilidades e competências que ele desenvolve ao longo da disciplina como um todo. Dessa forma, no momento que o aluno adquire uma nova habilidade ou demonstra conhecimento de um tópico ou assunto específico da disciplina, há um impacto direto sobre a sua nota, independente do período em que foi realizada a avaliação. Essa mecânica reforça a ideia da conquista do conhecimento.

Para atingir esses objetivos, a disciplina deixou de lado o sistema de notas tradicional (0 à 10), substituindo-o por um sistema de pontos de habilidade: todo o conteúdo da disciplina foi dividido em função dos temas abordados em cada unidade do semestre, como apresentado na Tabela 2.

A ideia é que cada exercício feito pelo aluno, seja através de listas ou provas da disciplina, implique na aquisição de pontos de habilidade - skills - em um ou mais tópicos listados na tabela. Desta forma, espera-se que o aluno tenha a sensação de que 
CBIE-LACLO 2015

Anais dos Workshops do IV Congresso Brasileiro de Informática na Educação (CBIE 2015)

\begin{tabular}{|c|c|c|}
\hline Habilidades & Pontos & Equivalente em Nota \\
\hline Lógica & 150 (I e III), 200 (II) & 1,5 (I e III), 2,0 (II) \\
\hline Variável & 100 & 1,0 \\
\hline Operadores & 150 & 1,5 \\
\hline Entrada/Saída & 100 & 1,0 \\
\hline Estruturas Condicionais & 200 & 2,0 \\
\hline Estruturas de Repetição & 300 & 3,0 \\
\hline Sub-rotinas & 200 & 2,0 \\
\hline Ponteiros & 200 & 2,0 \\
\hline Recursão & 200 & 2,0 \\
\hline Vetores & 200 & 2,0 \\
\hline String & 250 & 2,5 \\
\hline Matriz & 250 & 2,5 \\
\hline Registro & 250 & 2,5 \\
\hline Enumeração & 50 & 0,5 \\
\hline Arquivos & 50 & 0,5 \\
\hline
\end{tabular}

a cada atividade que realiza ele está galgando mais um degrau para adquirir uma nova habilidade e/ou conhecimento. No final, a nota da unidade será composta pelo progresso em cada um dos skills correspondentes. A partir de um determinado limiar de pontos (entre 100 a 250 pontos, dependendo do assunto) o aluno adquire aquela habilidade e a parte da nota referente à ela na composição da nota.

Pontos adquiridos em avaliações tem maior peso que pontos em exercícios. Uma vantagem dessa abordagem é que o aluno pode "compensar" um erro em uma prova através da execução de vários exercícios sobre o assunto específico. Por ter um peso menor que a avaliação, a quantidade de exercícios necessários para compensar uma questão de prova é bem maior, sendo necessário um real esforço e interesse por parte do aluno. A relação entre as pontuações é de 1 ponto/prova equivale a 3 pontos/exercício.

Outra preocupação para o andamento da disciplina são os grupos que dispõem de tempo limitado para estudo e exercícios ao longo do semestre, em especial os alunos do grupo All-Nighters. Para estimular esses alunos a investir o pouco tempo disponível em exercícios e atividades da disciplina, foi idealizado um segundo sistema de pontuação ligado a recompensas extrínsecas: basicamente o aluno terá a percepção de que, se dedicar tempo às atividades da disciplina, será diretamente recompensado com prêmios. Um dos objetivos desse sistema é prover um feedback rápido e palpável para o aluno que se esforçar nas tarefas da disciplina.

Para atingir esse objetivo, idealizou-se em um sistema de pontos de experiência $(x p)$, para que à medida que o aluno execute atividades e cumpra os objetivos estabelecidos, ele receba pontos associados à tarefa que renderão recompensas ao longo do semestre. Além de um prêmio pelo seu esforço, a recompensa representa também uma forma de mostrar para o aluno a sua progressão durante o curso. Todas as atividades que o aluno desempenha na disciplina (participação em aulas, exercícios feitos em casa, 


\begin{tabular}{|c|c|c|}
\hline Critério & Pontuação Unitária & Total \\
\hline Presença em aula & 1 xp/aula & $36 \mathrm{xp}$ \\
\hline Participação e interação com a turma & 1 xp/aula & $36 \times p$ \\
\hline Lista de exercícios & De 1 a 5 xp/questão & $400 \times p$ \\
\hline Avaliações & $250 \mathrm{xp} /$ prova & $750 \times p$ \\
\hline Exercícios em corretores online & 1 a 10 xp/questão & $800 \times p$ \\
\hline Total & & $2022 \times p$ \\
\hline
\end{tabular}

exercícios extras passados como desafios, atividades avaliativas) são pontuadas de forma que o aluno seja recompensado pela dedicação à disciplina. A experiência de uma atividade é determinada pela dificuldade e frequência em que ela ocorre. Logo, exercícios mais difíceis valem mais $x p$, enquanto atividades mais comuns (como participação em aula) valem uma quantidade menor de pontos.

As avaliações da disciplina são pensadas como desafios finais para cada unidade, refletindo nos sistemas de experiência e habilidades: as questões e itens avaliados em prova terão uma grande quantidade de pontos associadas, como se o aluno estivesse derrotando o adversário final de uma fase de jogo. Dessa forma, alunos que não quiserem participar da versão gamificada da disciplina não serão prejudicados no seu processo avaliativo, recaindo em uma metodologia tradicional.

O sistema de experiência foi concebido para ser pontuado através das atividades listadas na Tabela 3. Essa pontuação é adquirida pelos alunos e usada para progredir em um sistema de níveis. A cada passagem de nível, o aluno recebe uma recompensa que vai desde itens que ele pode utilizar na prova até prêmios tais como camisas e jogos.

A estrutura de níveis do aluno e das recompensas para cada nível é apresentada na Tabela 4. Dentre as recompensas possíveis, existem três categorias distintas:

- Itens de uso em prova

- XP Duplo: dobra o valor do XP de uma questão da prova;

- Troca de XP: troca o valor do XP de duas questões da prova;

- Bomba de Questão: ganha o XP de uma questão automaticamente.

- Itens de uso na disciplina (abono de faltas, ajuste de nota).

- Prêmios (alimentos, ingressos, jogos e camisas).

Durante a execução da disciplina, um esquema de feedback para os alunos é colocado em prática através de mensagens cadastradas em um sistema de turma virtual. Toda semana envia-se a lista com os dez primeiros lugares da turma, classificados pelo xp adquirido. Além disso, sempre que um aluno alcança uma recompensa, também é enviada uma mensagem individualizada parabenizando-o e informando o mesmo sobre os procedimentos necessários para receber e/ou usar a sua recompensa.

\section{Experimentação e Resultados Obtidos}

O modelo proposto está sendo validado em uma turma da disciplina de introdução às técnicas de programação de graduação em Tecnologia da Informação. Inicialmente, foi feito uso de sistemas já disponíveis para implementar os elementos do modelo, como o 
CBIE-LACLO 2015

Anais dos Workshops do IV Congresso Brasileiro de Informática na Educação (CBIE 2015)

\begin{tabular}{|c|c|c|}
\hline Nível & XP necessário & Recompensas \\
\hline 0 & $0 \times \mathrm{xp}$ & Nenhuma \\
\hline 1 & $60 \times p$ & Pipoca Bokus \\
\hline 2 & $130 \times p$ & Abono de Falta +1 \\
\hline 3 & $210 \times p$ & XP Duplo (1 uso) \\
\hline 4 & $300 \times p$ & Ajuda Nota +0.5 \\
\hline 5 & $400 \times p$ & Chocolate \\
\hline 6 & $510 \times p$ & Troca de XP (1 uso) \\
\hline 7 & $630 \times p$ & Falta +1 (todos) \\
\hline 8 & $760 \times p$ & Bomba de questão (1 uso) \\
\hline 9 & 900 xp & Ajuda Nota +0.5 \\
\hline 10 & $1050 \times p$ & Ingresso no cinema ( 1 inteira ou 2 meias) \\
\hline S & $1500 \mathrm{xp}$ & Jogo no Steam ou camiseta do Qwertee \\
\hline
\end{tabular}

ambiente virtual do próprio sistema acadêmico da instituição para permitir comunicação (tanto individual como coletiva) de informações e anúncio do ranking dos alunos. Os sistemas de experiências e habilidades foram modelados através de planilhas de dados interligadas, para realização do controle tanto da pontuação adquirida por cada aluno quanto das recompensas obtidas com a progressão dos níveis.

Ao final do curso, a maior parte da turma atingiu os níveis 6 e 7 de experiência. Alunos mais avançados conseguiram sua progressão até o nível 10. A média geral das notas foi de 6,13 com desvio padrão de 3,0, mais alta que a de semestres anteriores $(5,0$ com desvio padrão de 2,7). A taxa de aprovação foi de $65,9 \%$, bem acima da média de $32 \%$ dos últimos dois anos. Além disso, apenas $20,4 \%$ de alunos desistiram da disciplina comparado aos $49 \%$ de desistentes no semestre anterior. Estes resultados parciais são bastante positivos para o modelo proposto.

Ao final do semestre, um segundo questionário foi aplicado para obter um feedback dos alunos no que se refere a aplicação da metodologia, tendo sido respondido por 30 alunos. Cada ponto importante da metodologia (sistema de XP, Habilidades, Feedback) foi avaliado para identificarmos os seus pontos positivos e negativos. Além disso, buscou-se captar se houve um maior estímulo por parte dos alunos devido à metodologia, e quais dos elementos foram os responsáveis na motivação dos alunos.

$80 \%$ da turma respondeu através do questionário que a metodologia tem gerado um impacto positivo na disciplina. Dentre os pontos positivos elencados, o principal foi uma maior motivação para participar das aulas e realizar os exercícios. Isso permitiu uma maior interação aluno-professor, o que possibilitou a detecção de pontos falhos no aprendizado (tanto da turma em geral como também específico de cada aluno), ajustes na disciplina em tempo de execução e acompanhamento individualizado do progresso de cada estudante. O sistema de pontos de experiência foi apontado como um grande motivador nesse aspecto, porque a premiação era algo que os alunos gostavam de receber e utilizar durante a disciplina.

Além disso, os alunos indicaram que o sistema de habilidades reflete uma forma justa de avaliação contínua porque permite que o aluno se recupere ao longo do semestre. 


\section{CBIE-LACLO 2015}

Anais dos Workshops do IV Congresso Brasileiro de Informática na Educação (CBIE 2015)

Através das respostas dos questionários, muitos afirmaram que "se fosse uma disciplina tradicional, já teriam desisitido". A possibilidade de recuperar a nota demonstrando o aprendizado do conteúdo flexibiliza o processo de avaliação, adaptando-o para melhor acompanhar o ritmo de aprendizado de cada aluno.

Outro ponto positivo da metodologia foi a criação de um ambiente descontraído e confortável para os alunos, que passaram a se posicionar e criticar sem receios. Um exemplo disso aconteceu com a aplicação do elemento de Ranking: os alunos sentiram que a classificação semanal acrescentava uma característica de competitividade desnecessária à sala, sendo constrangedor para alunos que não conseguissem boas posições na classificação. O processo foi readaptado sem esse elemento, e apesar de ser necessário repetir a metodologia com outros grupos, existe um indício inicial de que o elemento de classificação não seja adequado para um ambiente de aula gamificado.

Dentre os aspectos negativos, destacou-se principalmente a falta de uma ferramenta que permitisse um acompanhamento individual dos pontos acumulados de forma mais prática e que permitisse um feedback imediato da situação de cada um com relação ao seu progresso no curso. A falta de integração de outras disciplinas do curso com essa metodologia foi outro ponto levantado, já que eles gostariam que as outras disciplinas adotassem metodologia similar à que está sendo desenvolvida na disciplina de programação.

\section{Considerações Finais}

A proposta aqui apresentada constitui uma primeira versão de modelo conceitual de sistema gamificado que está sendo experimentado em sala de aula.

Apesar de ainda estar em fase inicial, observou-se uma melhora promissora na motivação dos alunos e na forma como eles se integraram com as atividades da disciplina. Apesar de apresentar dificuldades com os assuntos, poucos alunos desistiram ao longo do semestre. A percepção da nota pelos alunos foi um ponto importante de trabalho da metodologia: a nota deixou de ser vista meramente como um número e passou a representar o esforço e domínio dos conteúdos da disciplina. Essa mudança na percepção fez com que alunos com baixo desempenho em avaliações conseguissem identificar os conteúdos deficientes e reforçar o seu estudo, não apenas para recuperar a nota, mas também para acompanhar a evolução dos assuntos ao longo do semestre.

Durante a execução percebeu-se a necessidade de um sistema personalizado para o controle e consulta dos pontos adquiridos pelos estudantes que viabilize à cada aluno a consulta dos seus registros de XP e habilidades a qualquer momento, melhorando o feedback do processo. O sistema também auxiliaria o professor da turma na gestão dos sistemas de pontos, recompensas dos alunos e entrega das atividades de forma individualizada. O próximo incremento do processo será o desenvolvimento dessa ferramenta, abrindo novas possibilidades de uso de elementos de jogos na disciplina (avatares customizados, novas formas de interação entre os alunos, modo colaborativo multijogador). Essa investigação será interessante no sentido de compreender quais elementos são adequados ou não para um processo de gamificação na educação.

A adesão inicial à resolução de exercícios ainda não atingiu às expectativas iniciais: 17 alunos dos 40 integrantes da turma tem entregado atividades com regularidade, enquanto o restante tem entregue de forma esporádica. É interessante ressaltar que dos 


\section{CBIE-LACLO 2015}

Anais dos Workshops do IV Congresso Brasileiro de Informática na Educação (CBIE 2015)

grupos de personas traçados, alunos do perfil All Nighters estão participando de forma ativa dos exercícios, uma das preocupações iniciais do trabalho. Ao final do processo, será feita uma avaliação da integração de cada um dos grupos de usuários, com o intuito de pensar novas mecânicas e elementos que atraiam grupos com menor aderência ao modelo de gamificação adotado.

\section{Referências}

Borges, S. d. S., Reis, H. M., Durelli, V. H., Bittencourt, I. I., Jaques, P. A., and Isotani, S. (2013). Gamificação aplicada à educação: Um mapeamento sistemático. In XXIV Simpósio Brasileiro de Informática na Educação.

Brown, T. (2010). Design Thinking: uma metodologia poderosa para decretar o fim da velhas ideias. Elsevier.

Cheong, C., Cheong, F., and Filippou, J. (2013). Quick quiz: A gamified approach for enhancing learning. In PACIS, page 206.

da Rocha Seixas, L., Gomes, A. S., Melo Filho, I. J., and Rodrigues, R. L. (2014). Gamificação como estratégia no engajamento de estudantes do ensino fundamental. In Anais do Simpósio Brasileiro de Informática na Educação, volume 25, pages 559568.

Denny, P. (2013). The effect of virtual achievements on student engagement. In Proceedings of the SIGCHI Conference on Human Factors in Computing Systems, pages 763-772. ACM.

Deterding, S., Dixon, D., Khaled, R., and Nacke, L. (2011). From game design elements to gamefulness: Defining "gamification". In Proceedings of the 15th International Academic MindTrek Conference: Envisioning Future Media Environments, MindTrek '11, pages 9-15, New York, NY, USA. ACM.

Domínguez, A., Saenz-de Navarrete, J., De-Marcos, L., Fernández-Sanz, L., Pagés, C., and Martínez-Herráiz, J.-J. (2013). Gamifying learning experiences: Practical implications and outcomes. Computers \& Education, 63:380-392.

Falcão, A. P., Leite, M. D., and Tenório, M. M. (2014). Ferramenta de apoio ao ensino presencial utilizando gamificação e design de jogos. In Anais do Simpósio Brasileiro de Informática na Educação, volume 25, pages 526-533.

Gomes, A., Henriques, J., and Mendes, A. (2008). Uma proposta para ajudar alunos com dificuldades na aprendizagem inicial de programação de computadores. Educação, Formação \& Tecnologias-ISSN 1646-933X, 1(1):93-103.

Hamari, J., Koivisto, J., and Sarsa, H. (2014). Does gamification work?-a literature review of empirical studies on gamification. In System Sciences (HICSS), 2014 47th Hawaii International Conference on, pages 3025-3034. IEEE.

Jenkins, T. (2001). The motivation of students of programming. In ACM SIGCSE Bulletin, volume 33, pages 53-56. ACM.

Schell, J. (2014). The Art of Game Design: A book of lenses. CRC Press.

Werbach, K. and Hunter, D. (2012). For the Win: How game thinking can revolutionize your business. Wharton Digital Press. 